American Journal of Infectious Diseases 4 (4): 237-243, 2008

ISSN 1553-6203

(C) 2008 Science Publications

\title{
Cefepime Monotherapy is as Effective as Ceftriaxone Plus Amikacin in Pediatric Patients with Cancer and High-Risk Febrile Neutropenia: A Randomized Comparison
}

\author{
${ }^{1,2}$ Carlos Alberto Pires Pereira, ${ }^{1}$ Antonio Sérgio Petrilli, \\ ${ }^{1}$ Fabianne Altruda Carlesse, ${ }^{1}$ Flávio Augusto Vercillo Luisi, \\ ${ }^{1}$ Kátia Verônica Torres Barros da Silva and ${ }^{1}$ Maria Lúcia de Martino Lee \\ ${ }^{1}$ Pediatric Oncology Institute, GRAACC/Universidade Federal de São Paulo, UNIFESP \\ ${ }^{2}$ Department of Infectious Diseases, Universidade Federal de São Paulo, Brazil
}

\begin{abstract}
The empirical use of antibiotic therapies is widely accepted in patients with fever and neutropenia during cancer chemotherapy. The use of intravenous monotherapy with broad-spectrum antibiotics in patients with high-risk of complications is an appropriate alternative. However, few data are available in pediatric patients. We conducted a prospective, randomized, open study in patients with lymphoma or leukemia who had fever and neutropenia during chemotherapy. Patients were randomized to receive cefepime (CFP) or ceftriaxone plus amikacin (CFT+AK). A total of 57 patients with 125 episodes of fever and neutropenia were evaluated (CFP, 62 and CFT + AK, 63 episodes). The mean neutrophil count at admission was 118.6 cells $\mathrm{mm}^{-3}$ (CFP) and 107 cells $\mathrm{mm}^{-3}(\mathrm{CFT}+\mathrm{AK})$. The mean duration of neutropenia was 9.0 days (CFP) and 8.0 days (CFT+AK). Analyzing only the first episodes of each patient, CFP treatment was successful in $65.5 \%$ of the episodes and CFT+AK were successful in $64.3 \%$. Overall rates of success with modification were 90\% (CFP) and 89\% (CFT+AK). No major treatment-emergent toxicity was reported. Monotherapy with CFP seems to be as effective and safe as the combination of CFT+AK for initial empirical therapy in children and adolescents with NF.
\end{abstract}

Key words: Febrile neutropenia, cefepime, high-risk, leukemia, lymphoma.

\section{INTRODUCTION}

Fever is the most prominent sign of infection in neutropenic patients and very often may be the only evidence of infection. The prompt initiation of empirical antibiotics in Febrile Neutropenic (FN) patients has been the most important advance in the management of these patients $s^{[1-3]}$.

Combination therapy with a beta-lactam and an aminoglycoside has been traditionally recommended for febrile episodes in high-risk neutropenic patients, but there is now evidence that monotherapy with broadspectrum cephalosporin such as ceftazidime, cefepime or carbapenem is as effective as combination therapy ${ }^{[4-8]}$. Monotherapy offers the advantages of decreased toxicity (mainly in patients treated with many nephrotoxic drugs), lower cost and easy administration when compared with multidrug regimens ${ }^{[5,9-12]}$.

Cefepime (CFP) is an extended spectrum fourth generation cephalosporin. It is active against a broad spectrum of gram-positive and gram-negative bacteria, including methicillin-sensitive $S$. aureus, alphahemolytic streptococci and some strains of P. aeruginos $a^{[13,14]}$. Recent reports showed that CFP is effective and safe for empiric treatment of FN pediatric patients $^{[6,15,16]}$. However, there are only limited studies comparing CFP monotherapy with combination therapies in children with cancer and $\mathrm{FN}^{[15]}$.

The aim of this study was to compare the efficacy and safety of monotherapy with CFP versus CFT+AK in children and adolescents with FN.

\section{MATERIALS AND METHODS}

This was a prospective randomized open study conducted at the Pediatric Oncology InstituteGRAACC-Federal University of São Paulo-Brazil. The Ethics Committee approved the protocol and written informed consent was obtained from each child's parents or legal guardian. The eligible populations were

Corresponding Author: Antonio Sérgio Petrilli, Instituto de Oncologia Pediátrica, Universidade Federal de São Paulo, Escola Paulista de Medicina, Rua Botucatu, 743 04023-062-São Paulo, SP, Brazil

Tel: +55 11 5080-8475 Fax: +55 $115080-8480$ 
children and adolescents (0-21 years) with acute leukemia and stage III and IV Hodgkin and nonHodgkin lymphomas (considered at high-risk for infectious complications) hospitalized with FN. Fever was defined as an axillary temperature above $38^{\circ} \mathrm{C}$ or three measurements between $37.5^{\circ} \mathrm{C}$ and $38^{\circ} \mathrm{C}$, at intervals of at least $4 \mathrm{~h}$, over a $24 \mathrm{~h}$ period. Neutropenia was defined as an absolute neutrophils count (ANC) below 500 cells $\mathrm{mm}^{-3}$, or between 500 and 1000 cells $\mathrm{mm}^{-3}$ before the nadir of chemotherapy. Exclusion criteria were: history of hypersensitivity to beta-lactamics, pregnancy or breastfeeding, hepatic dysfunction (total serum billirubins $>3$-fold the upper limit of normality) or liver enzymes (ALT/AST) >5fold the upper limit of normality) and renal insufficiency (creatinine level increased 50\% above the upper limit of normality for age), those who developed fever during transfusion of blood products, bone narrow transplantation and patients who had received antibiotics within 2 weeks of the study start.

Initial assessment: All the patients were assessed for their medical history and were submitted to a complete physical examination and to the following laboratory tests: complete blood cell count, electrolytes, liver and renal function, urinalysis, urine and blood cultures from catheters and peripheral veins. In addition, cultures of the presumptive site of infection in cases with skin and soft tissue infections, diarrhea, or any localized infection, chest and sinus X-rays were performed.

Randomization: All the patients who developed fever and neutropenia were randomly assigned to receive either CFP or CFT+AK. CFP was administered at a dose of $150 \mathrm{mg} \mathrm{kg}^{-1}$ day $^{-1} \mathrm{TID}, \mathrm{CFT}$ was given at the dose of $100 \mathrm{mg} \mathrm{kg}^{-1} \mathrm{day}^{-1} \mathrm{BID}$ and $\mathrm{AK}$ at $15 \mathrm{mg} \mathrm{kg}$ day $^{-1} \mathrm{QD}$, as intravenous infusion. The randomization was based on number lists and a patient could be randomized more than once if he/she had a distinct episode of $\mathrm{FN}$ and prior antibiotic treatment had been completed at least 2 weeks before.

Patients were evaluated daily by physical examination and complete blood count and weekly, for electrolytes, hepatic and renal function. Blood cultures were obtained each day, as long as the patient remained febrile. Chest X-rays were taken when clinically indicated.

Therapy was modified with the inclusion of new antibiotics, antifungal or antiviral agents, according to clinical status, development of clinically or microbiologically documented infections or persistence of fever. Amphotericin B was started when FN persisted for more than 5 days, or earlier, in case of suspected or documented fungal infection. Vancomycin was added when gram-positive cocci were isolated, when there was documented catheter related infection, skin infection or pulmonary infection, or in cases associated with hypotension. Antibiotics were discontinued after the second consecutive day without fever in patients with ANC $>500$ cells $\mathrm{mm}^{-3}$ without an identified source of infection. Patients were treated for a minimum of 5 days. Bacterial isolates were identified according to standard techniques and antibiotic susceptibilities were determined by disk diffusion, according to the National Committee for Clinical Laboratory Standards ${ }^{[17]}$. The $\mathrm{FN}$ episodes were classified at the end of the treatment period as (1) Microbiologically documented infection, including bacteremia (MDI), (2) Clinically Documented Infection (CDI) or (3) Fever of Unknown Origin (FUO) if no clinical or microbiological infection was identified. Clinical or MDI were treated for as long as necessary. At least 2 sets of positive blood cultures were required in case of infection with coagulase-negative Staphylococci $(\mathrm{CoN} S)$.

Diagnostic criteria and outcome: Therapeutic success was defined as resolution of all signs and symptoms without modification of the initial empirical antibacterial regimen, failure was defined as death due to infection, or the administration of any additional antibacterial agent due to persistent fever, persistent fever in a patient with signs of clinical deterioration, microbiological evidence, clinical progression of the presumed infection or adverse event associated with the antibiotic regime ${ }^{[18,19]}$. Fever was considered as an isolated cause of failure only after 7 days of treatment, or 2 days after the introduction of amphotericin B. We also used the definition of therapeutic success with modification if FN resolved with the addition of another antibiotic, antiviral or antifungal agent to the initial treatment. Breakthrough infection was defined as any infection occurring between $72 \mathrm{~h}$ after treatment start and one week after discontinuation of the antibiotic regime $^{[20]}$.

Data analysis: The Student's t-test was used to evaluate the difference between any two means (duration of neutropenia, duration of fever and age). The difference between proportions was used to categorize the febrile episodes (FUO, CDI or MDI). The Chi-square test with Yates correction was used to evaluate the difference in the gender distribution and treatment outcome. A p-value $<0.05$ was considered statistically significant. 


\section{RESULTS AND DISCUSSION}

From January 2000 to May 2002, 57 patients were included (29 in the CFP and 28 in the CFT+AK group) corresponding to a total of 130 episodes of FN. From the 57 patients, $22(38.6 \%)$ had one episode, $11(19.3 \%)$ had two, $16(28.1 \%)$ had three and 8 (14\%) had more than three episodes. Two episodes in the CFP group and 3 in CFT+AK group were excluded, because the ANC never fell below 500 cells $\mathrm{mm}^{-3}$. The study included, therefore, 62 and 63 episodes, respectively in the CFP and CFT+AK groups. The mean age of the patients treated with CFP was $8.9 \pm 4.9$ years (range 1 $18.0)$ and it was $8.9 \pm 4.8$ years (1.8-7.9) in the CFT+AK group. Table 1 shows demographic characteristics of the study patients and disease profile at inclusion. There was a higher prevalence of AML in the CFP group and of ALL in the CFT+AK group, but without statistic significance. In $67(53.6 \%)$ of the 125 episodes, an indwelling CVC was present. Of these, 31 (46.3\%) cases were randomized to CFP and $36(53.7 \%)$ to CFT+AK (Table 1).

The mean duration of fever was 3.9 days (1-13) and 4.4 days (1-14), respectively, in the CFP and $\mathrm{CFT}+\mathrm{AK}$ groups $(\mathrm{p}=\mathrm{NS})$. The mean duration of neutropenia was 9 days (2-27) and 8 days (2-15), respectively, in the CFP and CFT+AK groups ( $\mathrm{p}=\mathrm{NS}$ ) and the average time of treatment with antibiotics was 11.1 days (3-30) in the CFP group and 9.7 days (3-24) in the $\mathrm{CFT}+\mathrm{AK}$ group $(\mathrm{p}=\mathrm{NS})$.

Table 1: Demographic and baseline characteristics of patients by treatment groups

\begin{tabular}{lrrrr}
\hline & & & \multicolumn{2}{c}{ Ceftriaxone+ } \\
& Cefepime & \multicolumn{2}{c}{ Amikacin } \\
& \multicolumn{1}{c}{ N } & $(\%)$ & $\mathrm{N}$ & $(\%)$ \\
\hline Number of patients & 29.0 & 50.9 & 28.0 & 49.1 \\
Number of episodes & 62.0 & 49.6 & 63.0 & 50.4 \\
Episodes excluded & 2.0 & 1.5 & 3.0 & 2.3 \\
Gender & & & & \\
Female & 26.0 & 41.9 & 23.0 & 36.5 \\
Male & 36.0 & 58.1 & 40.0 & 63.5 \\
Race & & & & \\
Caucasian & 42.0 & 67.7 & 45.0 & 71.4 \\
Black & 20.0 & 32.3 & 17.0 & 27.0 \\
Others & 0.0 & & 1.0 & 1.6 \\
Neutrophil count average & 118.6 & & 107.0 & \\
(cells mm ${ }^{-3}$ ) & & & & \\
Underlying Diseases (UD) & & & & \\
ALL & 31.0 & 50.0 & 36.0 & 57.1 \\
AML & 23.0 & 37.1 & 16.0 & 25.4 \\
Non Hodgkin III & 3.0 & 4.8 & 6.0 & 9.5 \\
Non Hodgkin IV & 4.0 & 6.4 & 2.0 & 3.2 \\
Hodgkin Disease & 1.0 & 1.6 & 3.0 & 4.8 \\
Activity U D & & & & \\
Activity & 13.0 & 20.9 & 19.0 & 30.2 \\
Remission & 49.0 & 79.1 & 44.0 & 69.8 \\
Indwelling venous catheter & 31.0 & 46.3 & 36.0 & 53.7 \\
\hline
\end{tabular}

Fifty-four agents were isolated, thirty-seven in blood, 8 in urine and 8 from a catheter. CoNS, E. coli and Streptococcus sp. were the agents most frequently isolated. All gram-negative bacilli were susceptible to CFP, CFT and AK, except for one strain of $P$. aeruginosa (susceptible only to polimyxin B). Before $72 \mathrm{~h}$ of treatment, blood cultures were positive in $14.5 \%$ of the episodes for CFP and $14.3 \%$ for $\mathrm{CFT}+\mathrm{AK}$ and gram-positive bacteremia was predominant in both groups (55.6 and 66.7\%). After $72 \mathrm{~h}$ of treatment, blood cultures were positive in $9 / 62$ $(14.5 \%)$ and in 10/63 (15.9\%) patients in the CFP and $\mathrm{CFT}+\mathrm{AK}$ groups, respectively, with a predominance of gram-negative isolates $(60 \%)$ in the $\mathrm{CFT}+\mathrm{AK}$ group and gram-positives $(55.6 \%)$ in the CFP group. Considering all positive blood cultures, 23/37 (62.2\%) had gram-positives, 14/37 gram-negatives $(37.8 \%)$ and 3 were positive for fungi $(8.1 \%)$. The 3 fungal infections were isolated in the CFP group. In the overall analysis, the blood stream was considered as the site of infection in 18/62 (29\%) episodes for CFP patients and in $19 / 63(30.1 \%$ ) in the CFT+AK group (Table 2).

At the end of the treatment period, 51 episodes $(40.8 \%)$ were classified as CDI and $31(24.8 \%)$ as MDI, totaling $82(65.6 \%)$ episodes of documented infections in both groups. FOU occurred in 43 (34.4\%) episodes. There were no differences between the two groups. Breakthrough infections occurred in $22.6 \%$ (14/62) of the patients in the CFP group and in $15.9 \%(10 / 63)$ of those in the CFT+AK group ( $\mathrm{p}=\mathrm{NS})$ and were microbiologically documented in 3 episodes in each group.

Adverse events were reported in $22(17.6 \%)$ cases, 10 for CFP and 11 for CFT+AK. The main adverse events were diarrhea ( 1 case in each group), increased liver enzymes ( 3 cases in the CFT+AK group), headache ( 2 for CFP and 3 for CFT+AK) and increased creatinine ( 1 for CFP and 2 for CFT+AK ). All changes returned to normal after the end of the treatment.

The initial treatment was modified in $46(36.8 \%)$ cases, being $26(41.9 \%)$ in the CFP group and $20(31.7 \%)$ in the CFT+AK group $(\mathrm{p}=0.32)$. The most

Table 2: Pathogens recovered from 125 episodes of FN

\begin{tabular}{llllll}
\hline Agent & Blood & Catheter & Urine & Other & Total \\
\hline CoNS & 9 & 6 & 0 & 1 & 16 \\
E. coli & 6 & 1 & 6 & 0 & 13 \\
Streptococcus sp & 10 & 0 & 0 & 0 & 10 \\
Acinetobacter sp & 5 & 1 & 1 & 0 & 7 \\
P.aeruginosa & 2 & 0 & 0 & 0 & 2 \\
Other & 2 & 0 & 1 & 0 & 2 \\
Candida sp & 3 & 0 & 0 & 0 & 3 \\
Total & 37 & 8 & 8 & 1 & 54 \\
\hline
\end{tabular}

CoNS = Coagulase Negative Staphylococcus 
Table 3: Drug modification of initial therapy by study group

\begin{tabular}{llrrr}
\hline & Cefepime & \multicolumn{2}{c}{ Ceftriaxone+Amikacin } \\
& $-\mathrm{N}$ & $(\%)$ & \multicolumn{1}{c}{$\mathrm{N}$} & $(\%)$ \\
\hline Associated drugs & 16 & 26.0 & 10 & 16.0 \\
\hline Amphotericin B & 11 & 17.7 & 11 & 17.0 \\
Vancomycin & 3 & 4.8 & 5 & 7.9 \\
Clindamycin & 5 & 8.0 & 4 & 6.3 \\
Metronidazole & 8 & 13.0 & 0 & 0.0 \\
Amikacin & 7 & 11.0 & 7 & 11.0 \\
Other & 26 & 41.9 & 20 & 31.7 \\
Episodes with addition* & 26 & 100.0 & 63 & 100.0 \\
Total episodes & 62 & & &
\end{tabular}

Table 4: Overall response of the first episodes to initial therapy

\begin{tabular}{|c|c|c|c|c|}
\hline & \multicolumn{2}{|c|}{ Cefepime } & \multicolumn{2}{|c|}{$\begin{array}{l}\text { Cetriaxone+ } \\
\text { Amikacin }\end{array}$} \\
\hline & $\mathrm{N}$ & $(\%)$ & $\mathrm{N}$ & $(\%)$ \\
\hline Success & 19 & 65.5 & 18 & 64.3 \\
\hline With modification & 27 & 93.1 & 25 & 89.0 \\
\hline Failure & 11 & 34.5 & 10 & 35.7 \\
\hline Fever and clinical deterioration & 1 & 3.5 & 2 & 7.1 \\
\hline Fever without clinical deterioration & 4 & 13.8 & 3 & 10.3 \\
\hline Microbiological evidence & 3 & 10.3 & 3 & 10.3 \\
\hline Clinical progression of infection & 1 & 3.5 & 1 & 3.6 \\
\hline Adverse event & 0 & 0.0 & 0 & 0.0 \\
\hline Death & 1 & 3.5 & 1 & 3.6 \\
\hline Total & 29 & 100.0 & 28 & 100.0 \\
\hline
\end{tabular}

frequent drugs added, in both groups, were amphotericin B and vancomycin (Table 3).

Analyzing only the first episodes of each patient (29 in the CFP group and 28 for CFT+AK), as recommended by the Multinational Association for Supportive Care in Cancer, success was achieved in $19(65.53 \%)$ and $18(64.3 \%)$ and failure in $10(34.5 \%)$ and $10(35.7 \%)$ with CFP and CFT+AK, respectively. The main causes of failure in both groups were persistent fever without clinical deterioration and microbiological evidence. Success with modification occurred in $27(93.0 \%)$ and $25(89.0 \%)$ cases in the $\mathrm{CFP}$ and $\mathrm{CFT}+\mathrm{AK}$ groups, respectively. Analyzing all episodes, 3 cases (4.8\%) in the CFP group and $4(6.3 \%)$ in the $\mathrm{CFT}+\mathrm{AK}$ group required modification of the initial therapy and one child died in each group (Table 4).

Patients with acute leukemia and stage III and IV lymphomas have a higher risk of infectious complications ${ }^{[1,2,21-23]}$. The underlying disease and intensive chemotherapy lead to prolonged neutropenia, more frequent bacteremia, secondary infection and higher risk of death ${ }^{[24,25]}$.

The standard therapy for FN is a combination of antibiotics, which allows to treat a broad range of possible pathogens, achieves bactericidal serum concentrations, exerts a synergistic effect against some gram-negative bacilli and has a minimal risk of drug resistance during treatment ${ }^{[7,26]}$. However, with the worldwide decrease in the frequency of gram-negative infections in neutropenic patients and the availability of new antibiotics with extended spectrum of activity, the treatment of FN with a single antibiotic (monotherapy) is an alternative to combinations of beta-lactams plus aminoglycosides $^{[5,7,10,11,27,28]}$.

Because of its broad spectrum (including P. aeruginosa and gram-positive) and low toxicity, CFP is an excellent candidate for use as an empiric monotherapy ${ }^{[13,14,23]}$. Based on these features we prospectively randomized 57 high-risk patients with 125 episodes of FN. Considering the first episodes, the therapeutic success was similar $(65.5 \%$ vs $64.3 \%)$ in the $\mathrm{CFP}$ and $\mathrm{CFT}+\mathrm{AK}$ groups. The main causes of failure were persistent fever without clinical deterioration and microbiological evidence, in both groups. Analyzing all episodes, the success rate with modifications was $93.1 \%$ in $\mathrm{CFP}$ and $89 \%$ in the $\mathrm{CFT}+\mathrm{AK}$ group and mortality was around $3.5 \%$ (without statistical difference). Two meta-analyses compared the effectiveness of a beta-lactam monotherapy versus a beta-lactam-aminoglycoside combination for the treatment of FN patients as did the present study. One analyzed 47 randomized trials with 8,803 episodes and another, 29 randomized clinical trials with 4,795 episodes. Similar to our study, both metaanalyses concluded that monotherapy was as effective as aminoglycoside-containing combinations. However, both meta-analyses enrolled adults and children. In the first study, only eight trials included children ( 5 restricted to children < 16 years old), in the second study, the enrolment of patients younger than 14 years occurred in only four studies and three trials included exclusively patients with low-risk neutropenia (solid tumors and lymphoma). On the other hand, our study evaluated only children with high-risk neutropenia ${ }^{[7,28]}$. It is worth mentioning that both meta-analyses, as well as our study compared a new beta-lactam with an older one.

CFP was associated with unexpectedly higher allcause mortality at 30 days, as compared to other betalactam antibiotics in another meta-analysis covering 33 randomized trials. Four studies recruited only children. Mortality was also higher with CFP than with ceftazidime and equal to that of meropenem, even when the full recommended dose was used. We did not observe a higher mortality rate in the CFP group, although it must be noticed that we analyzed mortality at the end of the treatment and this was a small study. Regarding treatment, microbiological failure and the need for modifications, these were comparable in the present study and in the above mentioned metaanalysis $^{[8]}$. 
There is only one study conducted in children with FN treated with CFP as monotherapy and compared with an aminoglycoside-containing combination. In this study that compared CFP with ceftazidime plus AK, the success rates with unmodified therapy was $52 \%$ and $40 \%$, respectively ${ }^{[11]}$. The worse results in this study were due to a mandatory addition of a glycopeptide if fever persisted for more than three days. Our study used more strict criteria for the introduction of vancomycin.

Studies conducted in children and comparing CFP monotherapy with other beta-lactams as monotherapy (ceftazidime, meropenem or piperacillin/ tazobactam) are more frequent. The therapeutic success rate without modifications in these studies was similar to ours (60-70\%) in the CFP arm. However, different from those studies, we analyzed only patients with leukemia and lymphoma (high-risk), the other studies included almost $30 \%$ of patients with solid tumors, known to be at a lower risk and to have a higher success rate $^{[6,15,16,23,29,30]}$.

In the past decades, gram-positive pathogens have been isolated more frequently than gram-negatives in patients with FN. In our study, the agents most often isolated were also gram-positive cocci (50\%), considering both groups, with CoNS being the single most common agent isolated ${ }^{[23,31,32]}$. Regarding gramnegative bacilli, E. coli and Acinetobacter sp were the most frequently isolated agents. All isolates were susceptible to the antibiotics studied but one $P$. aeruginosa was only sensitive to Polimyxin B. Three specimens of Candida sp were isolated, all of them in the CFP group, where AML was more prevalent although without statistical significance. We had one death in each arm, one was caused by therapeutic failure in a patient with multiresistant $P$. aeruginosa and the other was due to progression of a pulmonary infection. .

The addition of another antimicrobial agent was necessary in 41.9 and $31.7 \%$ of the cases, in the CFP and $\mathrm{CFT}+\mathrm{AK}$ groups, respectively. The most frequently used drugs were amphotericin B and vancomycin, in agreement with previous studies ${ }^{[24,33]}$. These additions were not considered as unequivocal evidence of failure of the initial empiric regimen, but as a consequence of serious and prolonged neutropenia ${ }^{[12]}$. In our institution, the routine use of glycopeptides as empiric therapy is not recommended ${ }^{[27,34]}$. In this study, glycopeptides were added in only $17.7 \%$ of the episodes in the CFP group, in 7 of them a gram-positive pathogen only susceptible to vancomycin was recovered and in 4 there was clinical deterioration. In the CFT+AK group, there was indication for glycopeptides in $17.0 \%$ of the episodes, in 6 due to the isolation of a gram-positive pathogen and in 5 due to clinical deterioration. It is important to consider that an indwelling CVC was present in $53.6 \%$ of all episodes.

Combination therapies including aminoglycosides have been associated with a significant higher rate of adverse events, mainly nephrotoxicity ${ }^{[28]}$. Adverse events were reported in $17.6 \%$ of the patients in our study and were mainly related to the gastrointestinal tract. The drugs were well tolerated and no antimicrobial treatment had to be interrupted due to side effects. We did not observe incremental nephrotoxicity with the combination therapy, but our study evaluated a small number of patients ${ }^{[11]}$. In conclusion, Monotherapy with CFP is as successful and safe as the combination of CFT+AK. It should be considered an appropriate option for pediatric patients at high risk of infection. There was no major toxicity associated with the study drugs and the therapy was well tolerated.

\section{REFERENCES}

1. Pizzo, P.A., K.J. Robichaud and R.N. Wesley et al., 1982. Fever in the pediatric and young adult patient with cancer. A prospective study of 1001 episodes. Medicine (Baltimore), 61: 153-165.

2. Freifeld, A.G., J.W. Hathorn and P.A. Pizzo, 1993. Infectious Complications in the Paediatric Cancer Patient. In: Principles and Practice of Paediatric Oncology, 2nd Edn., Pizzo P.A. and Poplack Dg (Eds.). JP Lippincott, Philadelphia.

3. Sharma, A. and N. Lokeshwar, 2005. Febrile neutropenia in haematological malignancies. J. Postgrad. Med., 51: 42-48.

4. Pizzo, A., 1993. Management of fever in patients with cancer and treatment-induced neutropenia. N. Engl. J. Med., 328: 1323-1332.

5. Ramphal, R., 1999. Is monotherapy for febrile neutropenia still a viable alternative? Clin. Infect. Dis., 29: 508-514.

6. Chuang, Y.Y., I.J. Hung and C.P. Yang et al., 2002. Cefepime versus ceftazidime as empiric monotherapy for fever and neutropenia in children with cancer. Pediat. Infect. Dis. J., 21: 203-209.

7. Furno, P., G. Bucaneve and A. Del Favero, 2002. Monotherapy or aminoglycoside-containing combinations for empirical antibiotic treatment of febrile neutropenic patients: A meta-analysis. Lancet Infect. Dis., 2: 231-242.

8. Paul, M., D. Yahav, A. Fraser and L. Leibovici, 2006. Empirical antibiotic monotherapy for febrile neutropenia: Systematic review and meta-analysis of randomized controlled trials. J. Antimicrob. Chemother., 57: 176-189. 
9. Biron, P., C. Fuhrmann, H. Cure and P. Viens et al., 1998. Cefepime versus imipenemcilastatin as empirical monotherapy in 400 febrile patients with short duration neutropenia. CEMIC (Study Group of Infectious Diseases in Cancer). J. Antimicrob. Chemother., 42: 511-518.

10. Ramphal, R., R. Gucalp and C. Rotstein et al., 1996. Clinical experience with single agent and combination regimens in the management of infection in the febrile neutropenic patient. Am. J. Med., 1006: 83S-89S.

11. Corapcioglu, F. and N. Sarper, 2005. Cefepime versus ceftazidime + amikacin as empirical therapy for febrile neutropenia in children with cancer: A prospective randomized trial of the treatment efficacy and cost. Pediat. Hematol. Oncol., 22: $59-70$.

12. De Pauw, B.E., S.C. Deresinski and R. Feld et al., 1994. Ceftazidime compared with piperacillin and tobramycin for the empiric treatment of fever and neutropenic patients with cancer. A multicenter randomized trial. Intercontinental Antimicrobial Study Group. Ann. Intern. Med., 120: 834-844.

13. Sanders, C.C., 1993. Cefepime the next generation? Clin. Infect. Dis., 17: 369-379.

14. Eggimann, P., M.P. Glauser and M. Aoun et al., 1993. Cefepime monotherapy for the empirical treatment of fever in granulocytopenic cancer patients. J. Antimicrob. Chemother., 32: 151-163.

15. Mustafa, M.M., L. Carlson and I. Tkaczewski et al., 2001. Comparative study of cefepime versus ceftazidime in the empiric treatment of pediatric cancer patients with fever and neutropenia. Pediat. Infect. Dis. J., 20: 362-369.

16. Kebudi, R., O. Gorgun and I. Ayan et al., 2001. Randomized comparison of cefepime versus ceftazidime monotherapy for fever and neutropenia in children with solid tumors. Med. Pediat. Oncol., 36: 434-441.

17. Jorgensen, J.H., M.J. Ferraro and W.A. Craig, 1997. National Committee for Clinical Laboratory Standards. Performance Standards for Antimicrobial Susceptibility Tests. 6th Edn. Approved Standard. M2-A6,17:I, NCCLS, Pennsylvania.

18. The design, analysis and reporting of clinical trials on the empirical antibiotic management of the neutropenic patient-report of a consensus panelfrom the immunocopromised host society. J. Inf. Dis., 161: 397-401.
19. Feld, R., M. Paesmans and A.G. Freifeld et al., 2002. Immunocompromised host society, multinational association for supportive care in cancer, methodology for clinical trials involving patients with cancer who have febrile neutropenia: Updated guidelines of the Immunocompromised Host Society/Multinational Association For Supportive Care In Cancer, with emphasis on outpatient studies. Clin. Infect. Dis., 35: 14631468.

20. Nucci, M., N. Spector and A.P. Bueno et al., 1997. Risk factors and attributable mortality associated with superinfections in neutropenic patients with cancer. Clin. Infect. Dis., 24: 575-579.

21. Froland, S.S., 1984. Bacterial infections in the compromised host. Scand J. Infect. Dis. Suppl., 43: 7-16.

22. Klastersky, J., 1989. Empiric treatment of infection during granulocytopenia: A comprehensive approach. Infection, 17: 59-64.

23. Oguz, A., C. Karadeniz and E.C. Citak et al., 2006. Experience with cefepime versus meropenem as empiric monotherapy for neutropenia and fever in pediatric patients with solid tumors. Pediat. Hematol. Oncol., 23: 245-253.

24. Petrilli, A.S., R. Melaragno and K.V. Barros et al., 1993. Fever and neutropenia in children with cancer: A therapeutic approach related to the underlying disease. Pediat. Infect. Dis., 12: 916-921.

25. Petrilli, A.S., A. Bianchi and E. Kusano et al., 1993. Fever and granulocytopenia in children with cancer: A study of 299 episodes with two treatment protocols in Brazil. Med. Pediat. Oncol., 21: 356-361.

26. Del Favero, A., G. Bucaneve and F. Menichetti, 1995. Empiric monotherapy in neutropenia: A realistic goal? Scand J. Infect. Dis. Suppl., 96: 34-37.

27. Hughes, W.T., d. Armstrong and G.P. Bodey et al., 2002. guidelines for the use of antimicrobial agents in neutropenic patients with cancer. Clin. Infect. Dis., 34: 730-751.

28. Paul, M., K. Soares-Weiser and L. Leibovici, 2003. $\beta$ lactam monotherapy versus beta lactamaminoglycoside combination therapy for fever with neutropenia: Systematic review and meta-analysis. Br. Med. J., 326: 1111-1115.

29. Corapcioglu, F., N. Sarper and E. Zengin, 2006. Monotherapy with piperacillin/tazobactam versus cefepime as empirical therapy for febrile neutropenia in pediatric cancer patients: A randomized comparison. Pediat. Hematol. Oncol., 23: $177-186$. 
30. Hung, K.C., H.H. Chiu and Y.C. Tseng et al., 2003. Monotherapy with meropenem versus combination therapy with ceftazidime plus amikacin as empirical therapy for neutropenic fever in children with malignancy. J. Microbiol. Immunol. Infect., 36: 254-259.

31. Ramphal, R., 2004. Changes in the etiology of bacteremia in febrile neutropenic patients and the susceptibilities of the currently isolated pathogens. Clin. Infect. Dis., 39: S25-S31.

32. Wisplinghoff, H., H. Seifert and R.P. Wenzel et al., 2003. Current trends in the epidemiology of nosocomial bloodstream infections in patients with hematological malignancies and solid neoplasms in hospitals in the United States. Clin. Infect. Dis., 36: $1103-1110$.
33. Freifeld, A.G., T. Walsh and D. Marshall et al., 1995. Monotherapy for fever and neutropenia in cancer patients: A randomized comparison of ceftazidime versus imipenem. J. Clin. Oncol.,13: 165-176.

34. Cometta, A., W.V. Kern and R. De Bock et al., 2003. International Antimicrobial Therapy Group of the European Organization for Research Treatment of Cancer. Vancomycin versus placebo for treating persistent fever in patients with neutropenic cancer receiving piperacillintazobactam monotherapy. Clin. Infect. Dis., 37: 382-389. 